\title{
Real-time electrochemical detection of discrete adsorption events
}

\author{
Bernadette M. Quinn, Pieter G. van ’t Hof, Serge G. Lemay \\ Kavli Institute of Nanoscience, Delft University of Technology,
}

Lorentzweg 1, 2628 CJ Delft, The Netherlands

\section{Supporting Information}

\section{Additional Experimental Details}

$\mathrm{Au}$ microelectrodes were fabricated using standard lithographic methods. An insulating layer of polymethylmethacrylate (PMMA) was spin-coated onto a $50 \mathrm{~nm}$ Au film evaporated onto a standard $\mathrm{Si}$ wafer with a sticking layer of Cr. E-beam lithography was used to write a predefined electrode pattern in the PMMA, which was then developed with methyl-iosbutylketone. The resulting microelectrodes were recessed disk electrodes and the recession was determined by the thickness of the insulation layer. Individual electrodes were located using an optical microscope. A silicone elastometer (Sylgard 184, Dow Corning) fluid cell filled with the electrolyte solution was then positioned over the microelectrode. The cell volume was ca. $300 \mu \mathrm{l}$ with a $300 \mu \mathrm{m}$ opening at the lower end allowing the electrolyte to contact the electrode surface. A silver/silver chloride electrode (BAS) was placed in the cell and electrochemical measurements performed using a two-electrode arrangement.

Electrodes with radii in the nanometer range were prepared using the method proposed by Slevin et al. ${ }^{1}$ Briefly a $25 \mu \mathrm{m} \mathrm{Pt}$ wire was etched in $50 \%$ saturated $\mathrm{CaCl}_{2}$ solution to a sharp point. The sharpened tip was then coated with electrophoretic polymer layer ${ }^{2}$ and cured at $125^{\circ} \mathrm{C}$ for $10 \mathrm{mins}$. The polymer layer shrinks back from the tip during curing to expose the sharpened tip. The geometry is however not well defined and to overcome this, gold was deposited at the tip following the method proposed by Chen and Kucernak. ${ }^{3}$ The resulting Au electrode is larger than the underlying etched Pt electrode but is more stable and is expected to have a geometry between a hemisphere and a sphere. The apparent electrode radius $r_{\text {app }}$ was estimated from the diffusion limited current response $I_{\mathrm{d}}$ for the oxidation of ferrocene methanol ( $\mathrm{FcMeOH})$ using

$$
I_{\mathrm{d}}=2 \pi F D c r_{\text {app }}
$$

where $D$ is the diffusion coefficient and $c$ the bulk concentration of $\mathrm{FcMeOH}$. 


\section{Dependence of current step size on $r_{\mathrm{b}} / r_{\mathrm{e}}$}

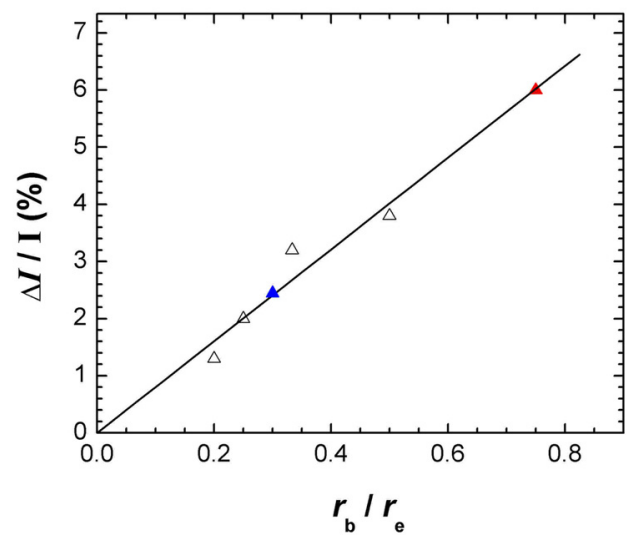

Figure S1. (a) Plot of relative current step values $(\Delta I / I)$ versus the ratio of microsphere to electrode radius $\left(r_{\mathrm{b}} / r_{\mathrm{e}}\right) . \Delta I$ refers to the average step decrease in current due to the interference of a single sphere on the oxidation current of a solution redox mediator $(I)$ at the microelectrode surface. The data was obtained from chronoamperometric measurements recorded at lithographically fabricated Au microelectrodes of varying radius $\left(r_{\mathrm{e}}\right)$ in the presence of $r_{\mathrm{b}}=0.5 \mu \mathrm{m}$ microspheres (open triangles) and $r_{\mathrm{b}}=0.15 \mu \mathrm{m}$ (filled blue triangle). The value obtained at a deposited Au nanoelectrode with $r_{\text {app }}=0.033 \mu \mathrm{m}$ in the presence of 0.025 $\mu \mathrm{m}$ spheres is also included (filled red triangle).

\section{Derivation of equation 1}

Equation 1 in the main text was derived as follows. The local bead flux $\boldsymbol{j}_{\text {mig }}$ due to migration at any position $\boldsymbol{r}$ in the bulk of the solution is given by ${ }^{4}$

$$
\boldsymbol{j}_{\text {mig }}(\boldsymbol{r})=u_{\text {bead }} n_{\text {bead }} \boldsymbol{E}(\boldsymbol{r}),
$$

where $\boldsymbol{E}(\boldsymbol{r})$ is the local electric field and $u_{\text {bead }}$ and $n_{\text {bead }}$ are the mobility and bulk number density of the beads, respectively. $\boldsymbol{E}(\boldsymbol{r})$ is in turn related to the total electrical current density $\boldsymbol{i}(\boldsymbol{r})$ by Ohm's law,

$$
\begin{aligned}
\boldsymbol{i}(\boldsymbol{r}) & =\boldsymbol{E}(\boldsymbol{r}) \sum_{m} z_{m} e n_{m} u_{m} \\
& \approx \boldsymbol{E}(\boldsymbol{r}) e n_{\mathrm{KCl}}\left(u_{\mathrm{K}^{+}}+u_{\mathrm{Cl}}\right) .
\end{aligned}
$$

In equation $\mathrm{S} 3$ the sum runs over all charged species in solution, with $e z_{m}, u_{m}$ and $n_{m}$ being the charge, mobility and number density of each species $m$. In practice in our experiments, this sum is completely dominated by the salt ions $\mathrm{K}^{+}$and $\mathrm{Cl}^{-}$since the bead concentration is sub-pM; this allows simplifying equation S3 to obtain equation S4.

The total rate of beads arriving at the electrode $J_{\text {mig }}$ and the total current $I$ are related to the local flux $\boldsymbol{j}_{\mathrm{mig}}$ and current density $\boldsymbol{i}$ by the linearity of Poisson's equation for the electrostatic field, yielding

$$
J_{\text {mig }} / I=\left|\boldsymbol{j}_{\text {mig }}\right| /|\boldsymbol{i}| .
$$

Combining equations S2, S4 and S5 yields equation 1 for the bead arrival rate at the electrode $J_{\text {mig. }}$. 


\section{Finite-element calculations}

The mass-transfer-limited transport of a neutral solution redox mediator to a disk electrode in the presence of one or several microspheres was determined by solving the diffusion equation, $\partial c(\boldsymbol{r}) / \partial t=D \nabla^{2}$ $c(\boldsymbol{r})$. Here $c(\boldsymbol{r})$ is the local concentration and $D$ is the diffusion constant of the mediator. The corresponding electrical current density is $n F D \nabla c(r)$, where $F$ is the Faraday constant and $n=1$ is the number of electrons transferred per molecule.

The diffusion equation was solved using finite-element methods (Femlab, Comsol AB, Stockholm) in two or three dimensions, as appropriate for each particular geometry. Steady-state solutions $(\partial c / \partial t=0)$ were obtained over a finite three-dimensional volume consisting of a hemisphere centered on the electrode. The outer boundary was sufficiently far from the electrode (5-10 times the electrode radius) that the diffusion profile was spherically symmetric at this boundary. This allowed matching the numerical solutions inside the simulated volume to the analytical solution for spherical diffusion outside this volume, permitting us to correct for the finite extent of the simulated volume.

The calculated dependence of the redox current on the position of a bead in the vicinity of the electrode surface is shown in figures S2 and S3. Figure S4 illustrates the effect that a bead has on the spatial distribution of the current density at the electrode surface.

The current was calculated to decrease by $35 \%$ for complete monolayer coverage assuming hexagonal close packing for $r_{\mathrm{b}} / r_{\mathrm{e}}=0.2$ for $Z / r_{\mathrm{b}}=0.125$.

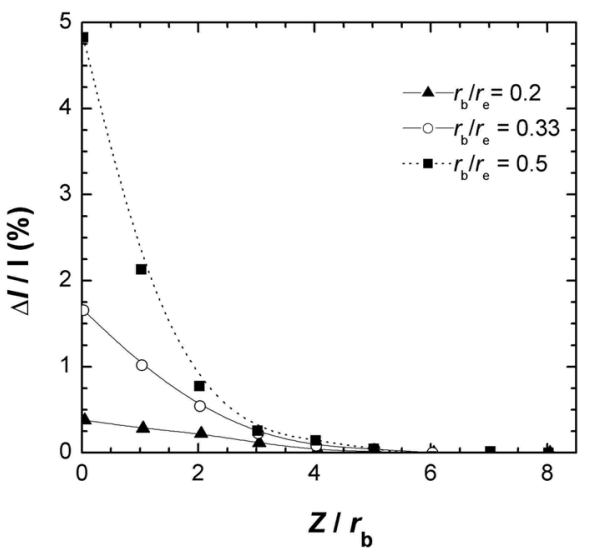

Figure $S 2$ Relative decrease in current $(\Delta I / I)$ at a microdisk electrode as a function of micropsheresurface distance $Z$ for three microsphere radii $r_{b}$. Here $Z$ is the distance from the microsphere surface to the electrode surface. In all cases, the microsphere approached the centre of the disk electrode.

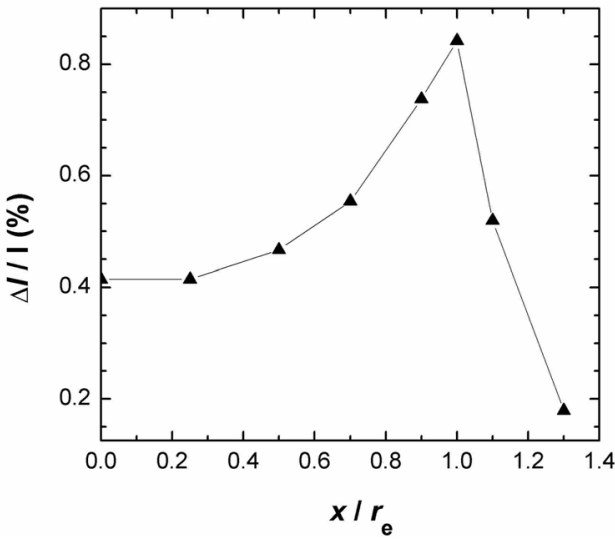

Figure $S 3$ Relative decrease in current $(\Delta I / I)$ at a microdisk electrode as a function of microsphere approach position to the electrode surface for $r_{\mathrm{b}} / r_{\mathrm{e}}=$ 0.2 where $x=0$ represents the origin of the disk and $x=1$ represents the disk edge. The microsphere is at a constant distance from the surface $z / r_{\mathrm{b}}=0.5$. The microsphere's influence on redox mediator current goes through a maximum as the sphere is moved from the center of the disk towards the edge. This is consistent with the higher current density at the extremities of the disk electrode. $\Delta I / I$ decreases for $x>1$ as the microsphere is no longer above the electrode surface and does not influence redox mediator flux for $x / r_{\mathrm{e}}>>1$. 
(a)

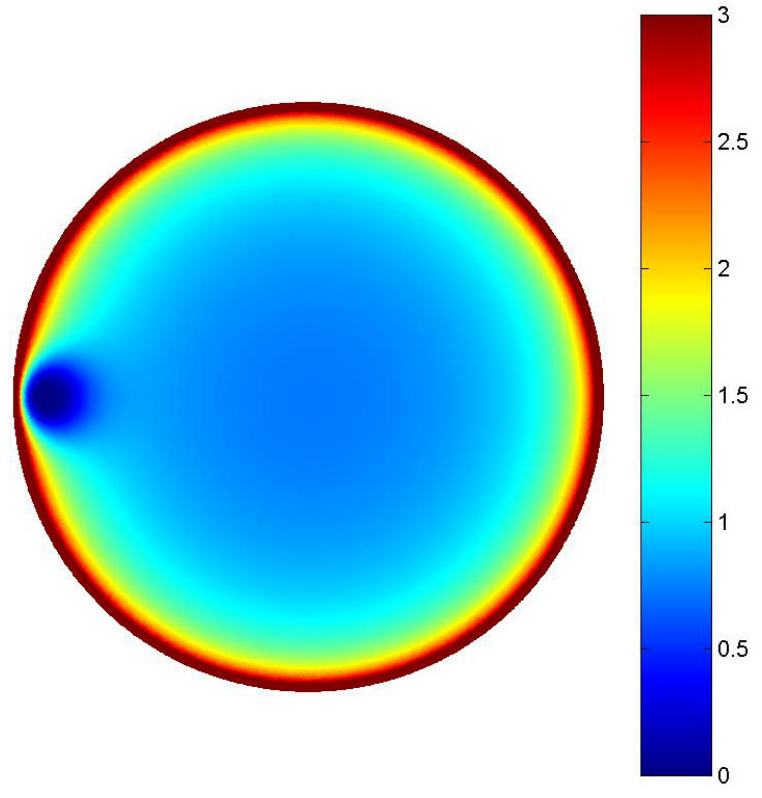

(b)

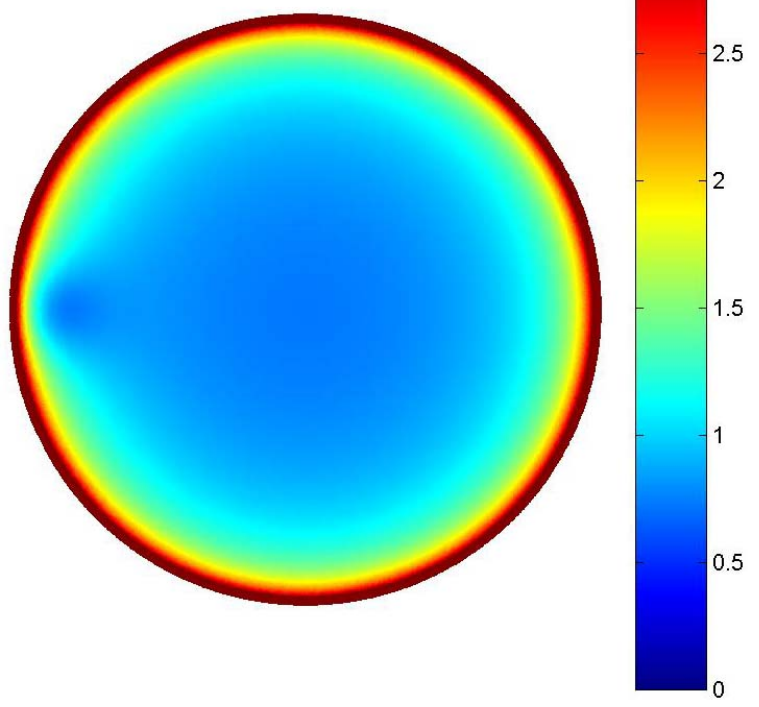

Figure S4 Surface image of the redox mediator current density at a disk electrode showing the influence of a single microsphere $\left(r_{\mathrm{b}} / r_{\mathrm{e}}=0.2\right)$ on the current density at $x / r_{\mathrm{b}}=0.9$ when the microsphere is very close to the electrode surface, $Z / r_{\mathrm{b}}=0.01$ (a) and one bead radius away from the surface, $Z / r_{\mathrm{b}}=1$ (b). The color bar shows that the current density on the electrode surface is highest at the disk edge and lowest at the centre of the disk. From (b), it can be clearly seen that the presence of the microsphere influences the current even when it is not in contact with the electrode surface.

\section{References}

(1) Slevin, C. J.; Gray, N. J.; MacPherson, J. V.; Webb, M. A.; Unwin, P. R. Electrochem. Commun. 1999, $1,282$.

(2) PPG, ZQ84-3225, Kind gift from Prof. H.S. White

(3) Chen, S. L.; Kucernak, A. J. Phys. Chem. B 2003, 107, 8392.

(4) Bard, A. J.; Faulkner, L. R. Electrochemical Methods, Fundamentals and Applications, 2nd ed.; John Wiley \& Sons: New York, 2001. 